\title{
Secular Trends in Long-Term Oral Bisphosphonate Use
}

\author{
Nathan Juergens, $M D^{7}$, Bruce Ettinger, $M D^{2}$, Rita Hui, PharmD, $M S^{3}$, \\ Malini Chandra, MS, MBA', and Joan C. LO, $M D^{1,2}$
}

'Department of Medicine, Kaiser Permanente Oakland Medical Center, Oakland, CA, USA; ${ }^{2}$ Division of Research, Kaiser Permanente Northern California, Oakland, CA, USA; ${ }^{3}$ Pharmacy Outcomes Research Group, Kaiser Permanente California, Oakland, CA, USA.

J Gen Intern Med 34(8):1383-4

DOI: $10.1007 / \mathrm{s} 11606-019-04934-7$

(C) Society of General Internal Medicine 2019

\section{INTRODUCTION}

Bisphosphonates (BP) have been used for over 20 years to treat osteoporosis, based on strong evidence of fracture risk reduction in high-risk women treated up to 5 years. ${ }^{1} \mathrm{Al}$ though discontinuation rates are high in the first year, greater treatment adherence is associated with increased fracture risk reduction. However, the optimal duration of BP therapy remains unclear. Since 2008, national declines in the number of patients receiving BP therapy have been observed, ${ }^{2,3}$ with total dispensed BP prescriptions falling from 31 to 14 million between 2008 and 2012. ${ }^{3}$ A confluence of factors may account for this, including concerns for rare adverse side effects ${ }^{2}$ and revised national practice guidelines reducing treatment of low-risk women ${ }^{4,5}$; these same factors may have prompted patients taking BP and providers prescribing BP to interrupt treatment. This report examines secular trends in long-term BP continuation and examines whether late non-adherence is more prominent in the years following new practice guidelines and news of potential harms from extended use.

\section{METHODS}

This study was conducted within Kaiser Permanente Northern California (KPNC) and approved by the KPNC Institutional Review Board. We identified women age $\geq 45$ years who initiated oral BP therapy (alendronate, risedronate, or ibandronate) during January 01, 2001, to December, 31, 2008 , excluding women without health plan membership in the 2 years prior or in the 5 years following BP initiation and those receiving intravenous BP during this period. Patient age (at BP initiation) and race/ethnicity were obtained from administrative records. Because the dominant non-white racial/ ethnic subgroup initiating BP was Asian, differences in race

Published online April 22, 2019 were compared among non-Hispanic white, Asian, and all others combined.

Using the days' supply of dispensed drug (excluding prescription overlap $>30$ days), with adherence calculated using the proportion of days covered, ${ }^{6}$ we identified those continuing adherent BP treatment for at least 3 and 5 years, defined by adherence $\geq 60 \%$ each year as well as BP treatment within the 60 days prior to the end of the treatment period. Continuation rates were examined by year of BP initiation. Demographic differences among women were examined by "year of initiation" (YOI) using the Student's $t$ test or chi-squared test. A $p$ value $<0.05$ was used as the threshold for statistical significance.

\section{RESULTS}

There were 49,268 women who initiated oral BP therapy during 2001-2008 and who met inclusion criteria. The average age was $68.4 \pm 10.1$ years; $66.4 \%$ were nonHispanic white, $18.1 \%$ Asian, and $15.5 \%$ of other or unknown race/ethnicity. Among these 49,268 women, 18,023 (36.6\%) and 11,810 (24.0\%) women continued adherent BP treatment for 3 and 5 years, respectively. The proportion with 5 -year adherent treatment was similar for women age $<65$ years $(23.5 \%)$ and $\geq 65$ years $(24.2 \%, p=0.08)$ at BP initiation. However, compared to non-Hispanic whites (23.4\%), Asian women showed greater 5-year adherence $(30.2 \%, p<0.001)$.

To evaluate secular trends in adherent continuation among those who initiated oral BP therapy, we examined data by YOI (Fig. 1). The proportion who adhered and continued treatment for at least 3 years was relatively stable for women with YOI 2001-2004 (37.5-40.6\%) but declined to $30.8 \%$ by YOI 2008 . The proportion with adherent treatment for 5 years was also relatively stable for YOI 2001-2004 (26.0-27.8\%) but declined to $17.3 \%$ by YOI 2008. The secular trend was comparable by age $\geq 65$ and age $<65$ years and by Asian or non-Hispanic white race/ethnicity (data not shown). When comparing BP YOI 2001-2004 and YOI 2005-2008 (Table 1), a significantly 


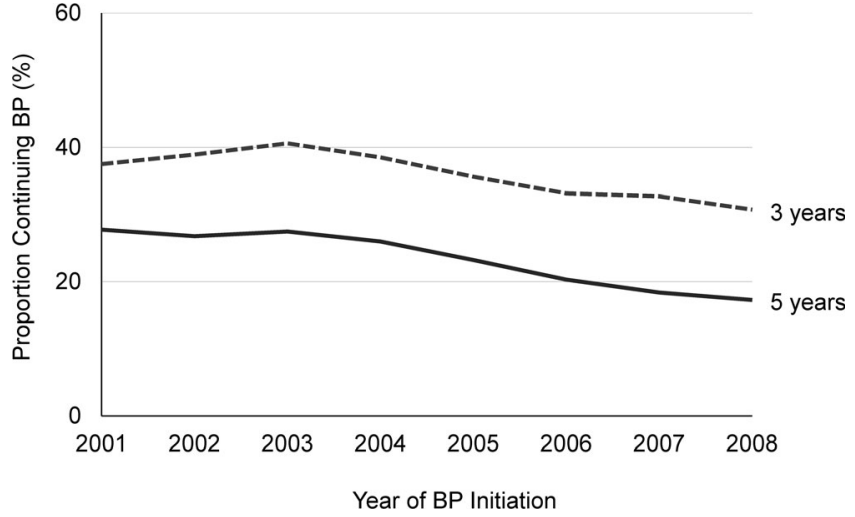

Figure 1 Proportion of women continuing adherent bisphosphonate (BP) treatment for 3 and 5 years by year of initiation.

lower proportion of women continued adherent BP treatment for 3 and 5 years after initiating BP in 2005-2008 $(p<0.05)$.

\section{DISCUSSION}

Temporal reductions in long-term BP continuation were seen among women initiating BP after 2004. This comports with changing practice guidelines and news reports of potential harm from long-term BP use promulgated within the past decade. While national use of BP drugs has declined by half since $2008,{ }^{3}$ and early discontinuation of BP has long been recognized, our data are the first to show that continuation of long-term BP therapy after treatment initiation has declined in recent years. Our current findings suggest the need for greater outreach and monitoring of adherence in women who initiate oral BP to ensure

Table 1 Women Initiating or Continuing Bisphosphonate (BP) Treatment by Year of Initiation (YOI)

\begin{tabular}{lll}
\hline \hline $\begin{array}{l}\text { Number initiating } \\
\text { BP treatment }\end{array}$ & $\begin{array}{l}\text { YOI 2001-2004 } \\
\boldsymbol{N}=\mathbf{2 8 , 1 7 9}\end{array}$ & $\begin{array}{l}\text { YOI 2005-2008 } \\
\mathbf{N}=\mathbf{2 1 , 0 8 9}\end{array}$ \\
\hline Race group & & $*$ \\
$\quad$ White & $19,124(67.9 \%)$ & $13,610(64.5 \%)$ \\
Asian & $4918(17.5 \%)$ & $3978(18.9 \%)$ \\
All others & $4137(14.7 \%)$ & $3501(16.6 \%)$ \\
Age at BP initiation & $67.8 \pm 10.0$ & $69.1 \pm 10.2 *$ \\
$\quad$ mean \pm SD) & & $*$ \\
Age at BP initiation & $10,783(38.3 \%)$ & $7217(34.2 \%)$ \\
$<65$ years & $9520(33.8 \%)$ & $6966(33.0 \%)$ \\
65-74 years & $7876(28.0 \%)$ & $6906(32.8 \%)$ \\
$\geq 75$ years & $11,019(39.1 \%)$ & $7004(33.2 \%)^{*}$ \\
Continuing adherent & & \\
treatment to 3 years after & & $4215(20.0 \%) *$ \\
BP initiation & $7595(27.0 \%)$ & \\
Continuing adherent & & \\
treatment to 5 years after & & \\
BP initiation & & \\
\hline
\end{tabular}

Continuing adherent treatment as defined by at least $60 \%$ BP adherence each year and treatment within 60 days prior to the end of the treatment period. Numbers represent $N(\%)$ unless otherwise indicated

$* p<0.05$ compared to 2001-2004 adequate treatment for the first 3-5 years. Whether the observed trends reflect patient non-adherence, cessation, or provider decisions to implement drug holidays is worthy of future study.

Corresponding Author: Joan C. Lo, MD; Division of Research, Kaiser Permanente Northern California, Oakland, CA, USA (e-mail: Joan.C.Lo@kp.org).

Funding This study received financial support from the Kaiser Permanente Northern California Community Benefit Grant and National Institute of Aging and National Institute of Arthritis, Musculoskeletal and Skin Diseases at the National Institutes of Health (RO1AG047230, S1).

\section{Compliance with Ethical Standards:}

This study was conducted within Kaiser Permanente Northern California (KPNC) and approved by the KPNC Institutional Review Board.

Conflict of Interest: The following conflicts of interest are reported: Joan Lo has received research funding from Amgen, Inc., and Sanofi Aventis and Malini Chandra has received research funding from Amgen, not pertaining to this study; Bruce Ettinger has served as an expert witness pertaining to litigation involving teriparatide (Teva Pharmaceuticals). The remaining authors have no conflicts of interest to disclose.

\section{REFERENCES}

1. Bilezikian JP. Efficacy of bisphosphonates in reducing fracture risk in postmenopausal osteoporosis. Am J Med. 2009;122:S14-21.

2. Jha S, Wang Z, Laucis $\mathbf{N}$, Bhattacharyya $\mathbf{T}$. Trends in media reports, oral bisphosphonate prescriptions and hip fractures 1996-2012: an ecological analysis. J Bone Miner Res. 2015;30:2179-87.

3. Wysowski DK, Greene P. Trends in osteoporosis treatment with oral and intravenous bisphosphonates in the United States, 2002-2012. Bone. 2013;57:423-8.

4. Clinician's Guide to Prevention and Treatment of Osteoporosis. Washington DC: National Osteoporosis Foundation; 2008.

5. Lee DR, Ettinger B, Chandra M, Hui RL, Lo JC. Changing patterns in oral bisphosphonate initiation in women between 2004 and 2012. J Am Geriatr Soc. 2017;65:656-8.

6. Hui RL, Adams AL, Niu F, et al. Predicting adherence and persistence with oral bisphosphonate therapy in an integrated health care delivery system. J Manag Care Spec Pharm. 2017;23:503-12.

Publisher's Note: Springer Nature remains neutral with regard to jurisdictional claims in published maps and institutional affiliations. 